\title{
A peck of Peppers
}

\author{
Independent studies introduced two distinct tools, both called 'Pepper', for live imaging of RNA.
}

R NAs are a crucial class of biomolecules whose roles extend to every aspect of cell biology. However, imaging them in live cells is challenging, as approaches for labeling RNAs that are compatible with living systems have lagged behind comparable tools for imaging proteins. One successful strategy for imaging RNA in living cells involves attaching multiple copies of an RNA tag to the RNA of interest, which in turn recruits many copies of a tagbinding protein fused to GFP to the tagged RNA. However, this strategy introduces a bulky label and may cause artifacts under some circumstances. An alternative strategy involves tagging RNAs of interest with one or more copies of an RNA aptamer that binds and activates a fluorogenic dye. In this case, the non-fluorescent dye is added to cells and only becomes fluorescent where the aptamer is present.

However, while existing tools like the 'vegetables' (Spinach, Broccoli and Corn), from Samie Jaffrey's laboratory at Weill Cornell Medical College, and others have been shown to be useful for imaging tagged mRNAs in mammalian cells, these tools suffer from some limitations in terms of folding and brightness that lessen their general utility, especially for imaging RNAs at the single-molecule level. To address these issues and to develop an alternative to the 'vegetables' that is fully genetically encoded, Wu et al. from the Jaffrey lab developed 'Pepper'.

Unlike the other vegetables, which combine RNA aptamers with various dyes, the Jaffrey lab's Pepper comprises an RNA hairpin known as the trans-activation response element (TAR) and a fluorescent protein (FP). The FP is destabilized by the bifunctional $\mathrm{tDeg}$ peptide tag, which combines a degron sequence with the Tat peptide. In cells where no Pepper RNA (TAR) is present, the tDeg-tagged FP is rapidly degraded. However, when present, the Pepper RNA binds the Tat peptide of the destabilized FP, blocking degradation. Thus, FP signal is present only when stabilized by the Pepper RNA. The researchers demonstrated that the Pepper RNA can stabilize several fluorescent and bioluminescent proteins, and that individual

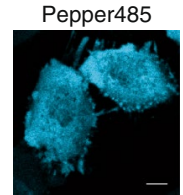

Pepper525

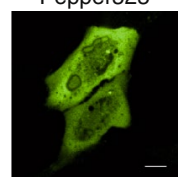

A rainbow of Peppers imaged in live mammalian cells. Figure adapted from Chen et al. https://doi.org/10.1038/s41587-019-0249-1 (Springer Nature America, Inc).

$\beta$-actin mRNAs in mammalian cells could be imaged when they harbor multiple copies of the Pepper RNA.

In parallel, Yi Yang's research group at East China University of Science and Technology developed an alternative set of Peppers for live imaging of RNA. Like the original 'vegetables', these Peppers are RNA aptamers that bind and activate fluorogenic dyes. Yang was motivated by his long-standing interest in developing intracellular sensors for metabolites. "Most current genetically encoded sensors are based on fluorescent proteins. However, highly responsive sensors are not only difficult to engineer, but are also limited in colors. In comparison, it is significantly easier to engineer highly responsive and colorful sensors based on fluorescent RNAs," notes Yang. However, as Yang and his team tried to use exiting RNA aptamers, they found themselves limited by brightness in mammalian cells. To make brighter RNA-based probes, Yang sought a close collaboration with Linlong Zhu, also at East China University of Science and Technology, who is an expert in dye development.

They developed their Pepper technology, which combines a series of eight related fluorogenic dyes ranging in color, from cyan to red, that can be bound and activated by their Pepper RNA for RNA-imaging in multiple colors. They demonstrate that each dye in combination with the Pepper RNA can be used for imaging RNA in live mammalian cells. They further demonstrate simultaneous imaging of red Pepper (Pepper620) and GFP for visualizing dCas9-GFP and sgRNA-Pepper in mammalian cells. Moreover, sgRNAs labeled with either Pepper620 or Broccoli could be expressed in the same cells to monitor multiple Cas9-sgRNA complexes.

In addition, the researchers showed they could quantify both BFP protein and mRNA levels in individual cells by tagging the BFP gene with a yellow Pepper (Pepper530). These studies revealed that different cell types have highly variable translation efficiencies - a result that surprised Yang. "We were most surprised that there were extremely weak correlations between protein and mRNA levels in a few cancer cell lines, which is rather counterintuitive. It would be interesting to further study whether Pepper-based single cell translation measurements could benefit cancer diagnosis or drug discovery," says Yang.

Despite the benefits of the Peppers, Yang notes there is room for improvement, and hopes that future efforts improve thermal stability and reduce magnesiumion-dependence of the aptamers. He also hopes to solve the structure and show that Peppers are useful for single-molecule imaging. Although the shared Pepper name may cause some confusion, it is clear that these Pepper technologies pave the way for improved RNA imaging in living cells.

Rita Strack

Editor's note: Rita Strack was a postdoctoral fellow in Samie Jaffrey's laboratory. She was not involved in the featured research.

Published online: 31 October 2019 https://doi.org/10.1038/s41592-019-0633-2

Research papers

$\mathrm{Wu}$, J. et al. Live imaging of mRNA using RNA-stabilized fluorogenic proteins. Nat. Meth. 16, 863-865 (2019).

Chen, X. et al. Visualizing RNA dynamics in live cells with bright and stable fluorescent RNAs. Nat. Biotechnol. https://doi.org/10.1038/s41587-0190249-1 (2019). 